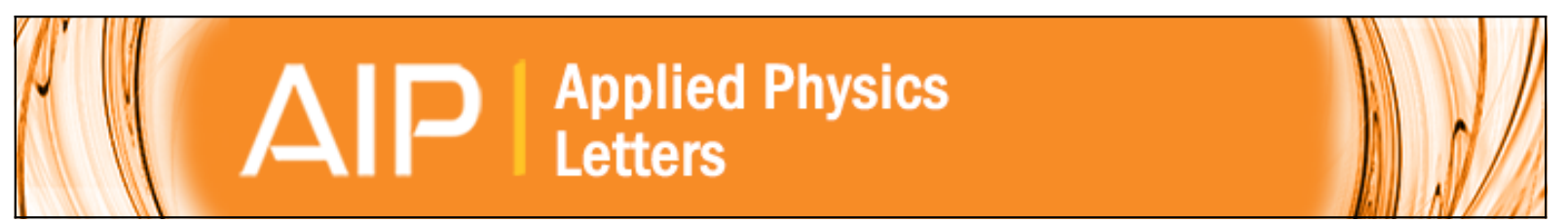

Using the fringing electric field in microfluidic volume sensors to enhance sensitivity and accuracy

Jason Riordon, Nicolas M.-Catafard, and Michel Godin

Citation: Applied Physics Letters 101, 154105 (2012); doi: 10.1063/1.4759033

View online: http://dx.doi.org/10.1063/1.4759033

View Table of Contents: http://scitation.aip.org/content/aip/journal/apl/101/15?ver=pdfcov

Published by the AIP Publishing

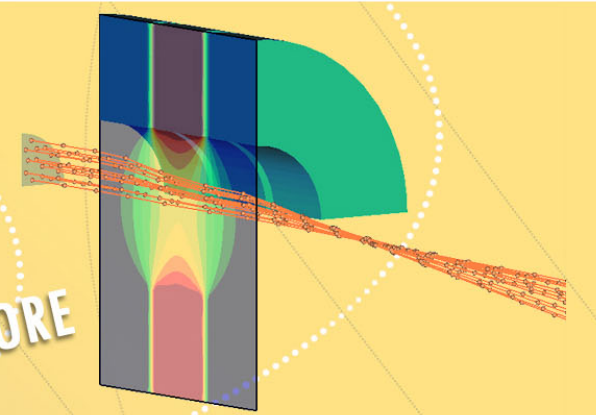




\title{
Using the fringing electric field in microfluidic volume sensors to enhance sensitivity and accuracy
}

\author{
Jason Riordon, Nicolas M.-Catafard, and Michel Godin \\ Department of Physics, University of Ottawa, Ottawa, Ontario K1N 6N5, Canada
}

(Received 20 August 2012; accepted 1 October 2012; published online 10 October 2012)

\begin{abstract}
The particle trajectory above impedance-monitoring coplanar electrodes in a microfluidic channel dramatically influences the measured electric current change. We use finite element modeling to predict changes in ionic current for microspheres flowing in highly fringing fields, and validate these results by introducing a buoyancy-based particle focusing technique. Using $6 \mu \mathrm{m}$ polystyrene particles in solutions of varying density, we control the height of the particle trajectories near the sensing electrodes and show that sensitivity can be increased by up to $3.5 \times$ when particles flow close to the electrodes compared to particles flowing further away, while simultaneously improving accuracy. (C) 2012 American Institute of Physics. [http://dx.doi.org/10.1063/1.4759033]
\end{abstract}

The use of microfluidic impedance-based flow cytometers has seen a great deal of growth in the past decade, providing an inexpensive means for detection of micron-sized particles with low sample consumption. Impedance-based volume sensors and micro-Coulter counters measure the change in ionic current as individual microtargets transit through a narrow sensing channel. ${ }^{1}$ Sensors have been developed to measure the volume of synthetic particles as well as a number of biotargets, such as red blood cells, ${ }^{2}$ yeast, ${ }^{3}$ MDCK (kidney) epithelial cells, ${ }^{4}$ and Escherichia coli. ${ }^{5}$ Multiple electrode configurations have been demonstrated, including parallel ${ }^{5}$ and planar ${ }^{6}$ electrode designs. While the latter is advantageous for ease of fabrication, it suffers from sensitivity to particle height, due to the electric field gradient. ${ }^{7}$ Consequently, microparticles that flow through the sensor along a path far from the electrodes induce a smaller change in resistance than those flowing nearest to the electrodes. $^{2}$ This adds uncertainty to the determination of particle volume, limiting the usefulness of coplanar electrode geometries in comparison with parallel electrodes. In this Letter, we use finite element method (FEM) modelling to study the current change response of microspheres flowing along trajectories at different heights within the sensor, and obtain a volume-dependant enhancement factor. To validate results experimentally, we introduce a buoyancy-based technique capable of focusing particles along trajectories at different vertical distances from the coplanar electrodes.

FEM modelling was performed using the COMSOL MULTIPHYSICs Conductive Media DC module. A $20 \mu \mathrm{m}$ microchannel of square cross section was modelled, with a pair of $20 \mu \mathrm{m}$ wide coplanar electrodes separated by a $20 \mu \mathrm{m}$ gap located on the top surface of the microchannel. A $30 \mathrm{mV}$ DC potential was simulated between both electrodes, drawing a $420 \mathrm{nA}$ current through conductive media $\left(\sigma=1.3 \Omega^{-1}\right.$ $\mathrm{m}^{-1}$ ). Current change was computed by positioning a nonconductive microsphere at fixed coordinates within the channel, and iterating over multiple configurations (Fig. 1). Every iteration required a separate FEM calculation using 169000 mesh elements. Electric current was obtained by integrating the normal electric field over the entire channel cross-section at a given position between the electrodes. To provide an estimate on uncertainty, four such cross sections were integrated. Error bars were smaller than the symbols in Fig. 1 and thus were omitted. Fig. 1(a) illustrates how the current pulse differs for microspheres flowing at different heights. The $\mathrm{x}$-axis shows the position with respect to the center of the electrode pair. For a microsphere flowing near the electrodes (Top), the maximum current change occurs as it goes by the electrode edge; for microspheres in the middle or at the bottom of the channel, maximum current change occurs at the center of the electrode pair. Fig. 1(b) maps the enhancement factor between the Bottom and Top configurations as a function of target volume (diameter on top axis). As shown in Fig. 1(a), this position of maximum change is different for each trajectory. The enhancement factor is greater for smaller microtargets than for larger ones: in the case of $6 \mu \mathrm{m}$ diameter microspheres, this corresponds to a $3.3 \times$ enhancement in signal.

A target's effect on the fringing electric field can be better visualised in Figs. 1(c) and 1(d), where a logarithmic plot of the electric field density is shown for a 2D slice of the system in the Top and Bottom configurations. As the nonconductive microsphere nears the inner corners of the electrodes (Fig. 1(c)), electric field perturbation is maximized leading to a larger change in current. A similar electric field fringing effect was also described in FEM simulations done by Gawad et al. with respect to parallel facing electrodes. ${ }^{8}$

To validate these simulations experimentally, $6 \mu \mathrm{m}$ diameter microspheres were flowed through a microfluidic volume sensor at different heights. This particle size was chosen so as to be small enough to see a significant enhancement between flow paths, and large enough to produce a large current change pulse. Flow height control was accomplished by altering glycerol concentration in the carrier buffer to control density, and thus generate a negative or positive buoyant force on the microparticles. The microfluidic volume sensor chip was fabricated by polydimethylsiloxane (PDMS) molding using photolithography. ${ }^{9}$ SU-8 10 photoresist (Microchem) was patterned on a silicon wafer to create a $20 \mu \mathrm{m}$ wide sensing channel of square cross-section. PDMS of 10:1 base:curing agent ratio was then molded, and bonded to a glass substrate with $\mathrm{Au} / \mathrm{Ti}$ electrodes $(80 \mathrm{~nm} / 5 \mathrm{~nm})$. The 
a)

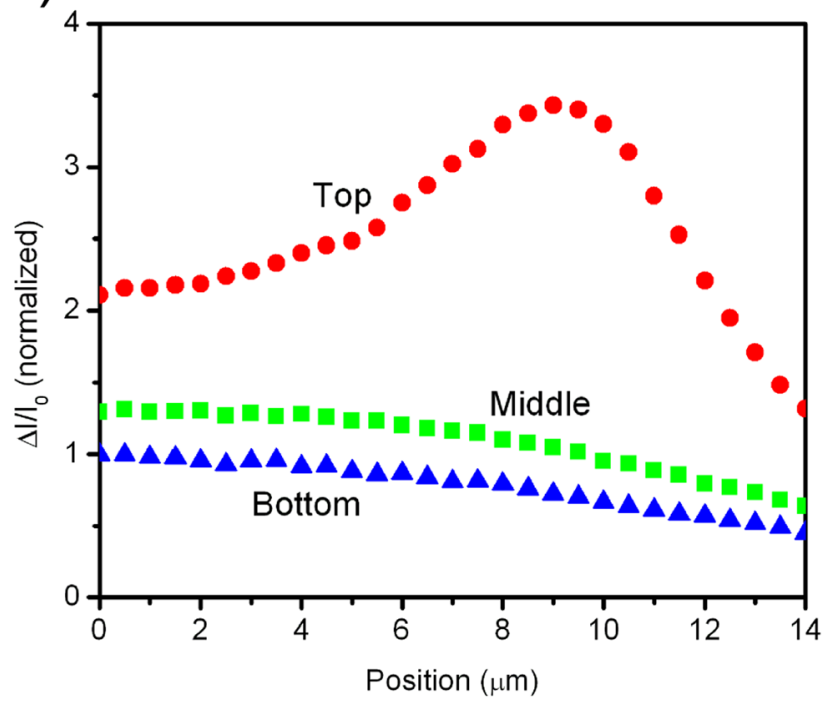

b)

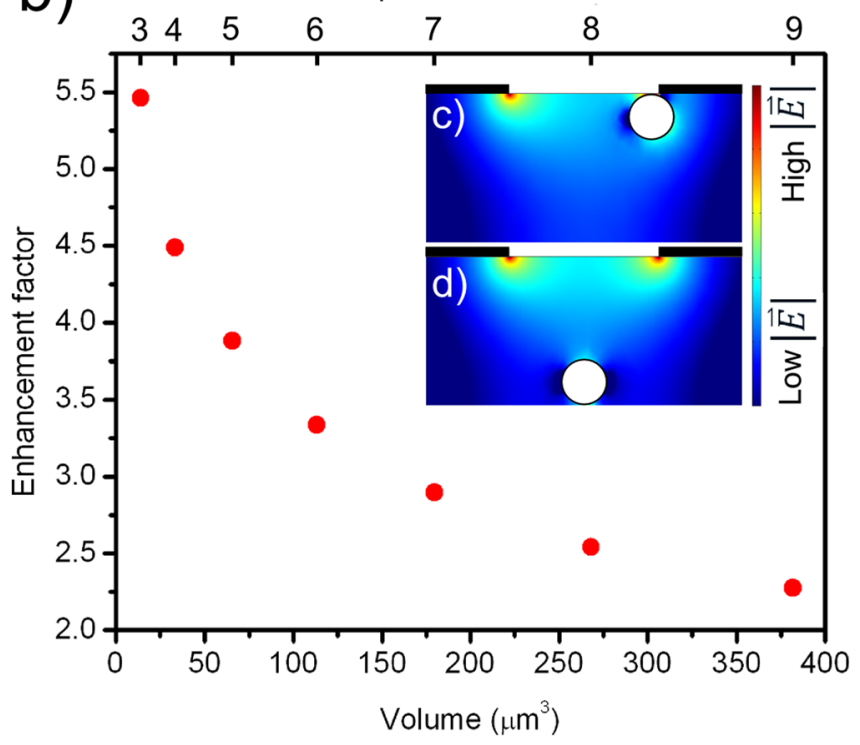

FIG. 1. (a) Current change for $d=6 \mu \mathrm{m}$ microspheres transiting a $20 \mu \mathrm{m}$ by $20 \mu \mathrm{m}$ sensing channel at different heights: Bottom $z=3.1 \mu \mathrm{m}$, Middle $z=10 \mu \mathrm{m}$, and Top $z=16.815 \mu \mathrm{m}$, each measured from the channel floor to the center of the microsphere. These positions were chosen so as to be at no more than $100 \mathrm{~nm}$ from the electrodes, which are positioned at the very top of the channel. The x-axis represents the position of the bead along the length of the channel, starting from the mid-point between the electrodes. For microspheres passing closest to the electrodes, the maximum current change occurs near the inner electrode edges $(x= \pm 10 \mu \mathrm{m})$. (b) Enhancement factor between Top and Bottom configurations for different microsphere volumes. (c) and (d) illustrate the electric field density in a 2D slice of the device (log plot) for a $6 \mu \mathrm{m}$ diameter microsphere in Top and Bottom configurations, respectively. The horizontal black bars illustrate electrode positioning.

electrodes were fabricated using a lift-off technique described elsewhere. ${ }^{9}$ The device is positioned under an upright microscope with the electrodes on glass at the top. The sensing channel connects two parallel bypass channels (Fig. 2(a)) and is designed to be of sufficient length to allow for targets to settle before transiting the sensor $(\sim 12 \mathrm{~mm}$ from inlet to sensor). From Stoke's Law, the terminal velocity, $v_{s}$, of suspended microspheres is proportional to the difference between the particle mass density $\rho_{p}$ and fluidic mass density $\rho_{f}$ a)

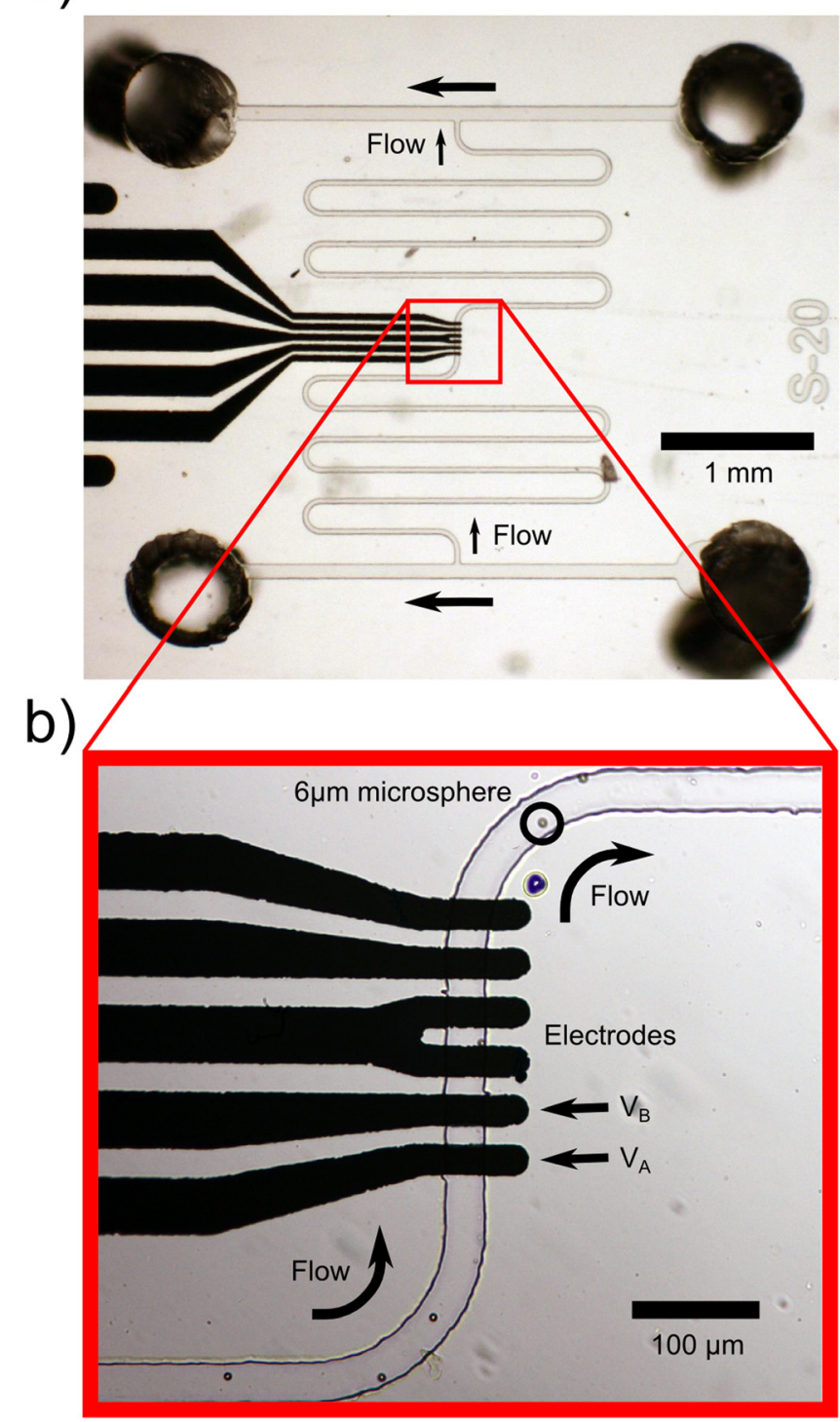

FIG. 2. (a) Microfluidic volume sensor with meandering channel connecting two parallel bypass channels. Au electrodes are positioned at the top of the channel. Arrows denote flow direction. (b) Enlarged view of sensing volume framed by two electrodes, where an $\mathrm{AC}$ potential is applied at $\mathrm{V}_{\mathrm{A}}$ and $\mathrm{V}_{\mathrm{B}}$ is grounded. Only the lower two electrodes are utilized in the experiments described here.

$$
v_{s}=\frac{2}{9} \frac{\left(\rho_{p}-\rho_{f}\right)}{\mu} g R^{2},
$$

where $\mu$ is the dynamic viscosity, $g$ the gravitational acceleration, and $R$ the microsphere radius. For $6 \mu \mathrm{m}$ diameter polystyrene microspheres (Bangs Labs) in a solution of glycerol of concentration up to $42 \%$, this leads to an upward velocity of $v \approx 0.3 \mu \mathrm{m} / \mathrm{s}$. For the device depicted in Fig. 2, this means maintaining a flow rate below $240 \mu \mathrm{m} / \mathrm{s}$ to insure complete microsphere migration to the electrodes. This serves as an upper estimate, since settling time is slightly increased due to wall effects.

Ionic current was monitored by applying a $50 \mathrm{kHz}$ signal $\left(\mathrm{V}_{\mathrm{pp}}=620 \mathrm{mV}\right)$ between the pair of sensing electrodes, and measuring current in series via a current amplifier (Keithley 428) and lock-in amplifier (Stanford Research Systems SR830 DSP). The probe signal is applied via a function 
generator (Stanford Research Systems DS345) and a lownoise preamplifier (Stanford Research Systems SR560) operating at unity gain. Lock-in amplitude is measured through a $200 \mathrm{kHz}$ 16-bit DAQ card (NI-USB 6353) using a LabView script. Data are filtered using a combination of point-bypoint averaging and low pass filtering. Flow through the channels was controlled by using pressure regulators (SMC IR2000-N02-R and SMC IR2010-N02-R) to establish the flow pattern in Fig. 2(a) as previously described. ${ }^{9}$ Three solutions with suspended $6 \mu \mathrm{m}$ polystyrene microspheres were prepared at an approximate concentration between $1 \times 10^{5}$ and $1 \times 10^{6}$ microspheres $/ \mathrm{ml}$. Solution densities were chosen to affect particle buoyancy, such as to draw microspheres up towards the sensing electrodes or down away from the electrodes. The difference in densities between that of the particle and of the carrier fluid was varied to control gravitational settling times. Each buffer contained $0.1 \%$ bovine serum albumin (BSA), $0.1 \mathrm{M} \mathrm{KCl}$, and varying concentrations of glycerol $(0 \%, 21 \%$, or $42 \%)$. Carrier fluid densities were quantified with a suspended microchannel resonator (SMR): ${ }^{10} 1.004 \pm 0.001 \mathrm{~g} / \mathrm{cm}^{3}, 1.065 \pm 0.001 \mathrm{~g} / \mathrm{cm}^{3}$, and $1.121 \pm 0.001 \mathrm{~g} / \mathrm{cm}^{3}$ for $0 \%, 21 \%$, and $42 \%$ glycerol solutions, respectively. To prevent beads from non-specifically interacting with microchannel surfaces, $0.1 \% \mathrm{~g} / \mathrm{ml}$ of detergent (Contrex, Decon Labs Inc.) was added prior to experimentation to both the water and glycerol heavy solutions.

For each solution, microspheres were flowed through the volume sensor at $\sim 100 \mu \mathrm{m} / \mathrm{s}$, and current changes were recorded. This speed was chosen to insure that transiting particles have enough time to settle by gravity as dictated by Eq. (1). Typical events are presented in Fig. 3(a). Microspheres flowing near the bottom of the channel (A-away from the sensing electrodes) cause a much lower change in current than those flowing at the top (D-near the sensing electrodes). Events shown in B and C are for particles suspended in a $21 \%$ glycerol solution, making them practically neutrally buoyant. The $21 \%$ glycerol solution has a density of $1.065 \mathrm{~g} / \mathrm{cm}^{3}$, which only slightly differs from that of the microspheres $\left(1.062 \mathrm{~g} / \mathrm{cm}^{3}\right)$. This small difference in densities is sufficient to cause a slight settling along the vertical axis in the timeframe of the experiment. In turning the device upside down, this shift is reversed (experiment B in Fig. 3(a)). Population histograms for respective samples are presented in Fig. 3(b). Current changes of $0.16 \% \pm 0.01 \%$, $0.26 \% \pm 0.01 \%, 0.35 \% \pm 0.03 \%$, and $0.55 \% \pm 0.05 \%$ were measured for experiments $\mathrm{A}, \mathrm{B}, \mathrm{C}$, and $\mathrm{D}$, respectively. There is a nearly 3.5 -fold increase in sensitivity when the particles assume a trajectory near the electrodes compared to when they flow near the bottom of the microchannel, as predicted from Fig. 1(b). One would expect beads flowing in a near-neutrally buoyant solution to follow a Poiseuille distribution, creating a histogram that would span the entire region between histograms $\mathrm{A}$ and $\mathrm{D}$ (from $\Delta \mathrm{I} / \mathrm{I}_{0}=0.16$ to 0.55). This is not the case, as the $\mathrm{B}$ and $\mathrm{C}$ distributions in Fig. 3(b) are too narrow. This suggests that targets are predisposed to follow a certain trajectory, likely due to the geometry of the device, and how microtargets are flowed into the sensor. Though experiments $\mathrm{B}$ and $\mathrm{C}$ produced a mean current change of similar uncertainty to experiments $\mathrm{A}$ and $\mathrm{D}$, their accuracy differs greatly.
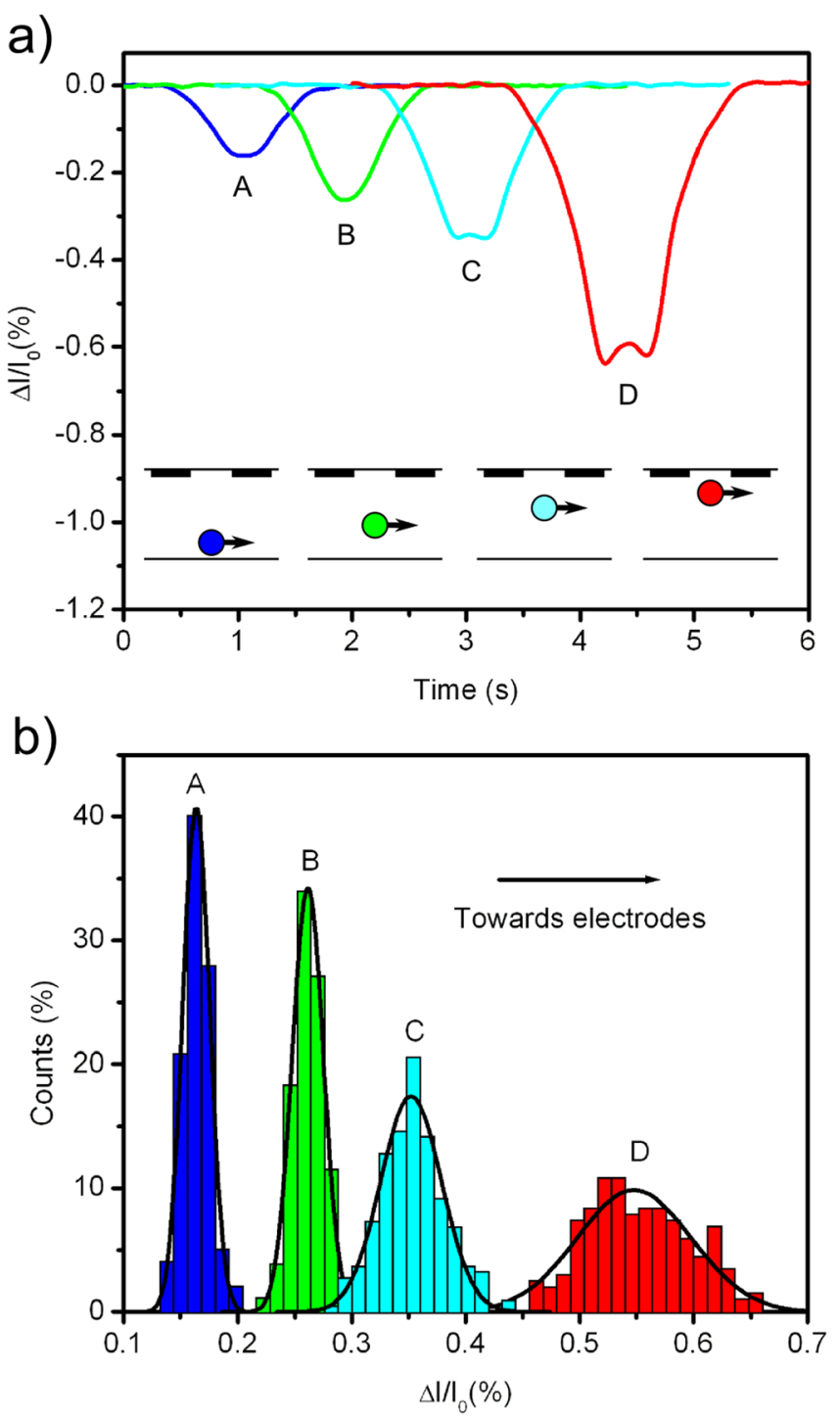

FIG. 3. (a) Typical current change for a $6 \mu \mathrm{m}$ diameter polystyrene microsphere flowing through a $20 \mu \mathrm{m}$ wide square channel for 4 experiments with three different solutions of varying density: $\mathrm{A}=0 \%, \mathrm{~B}=\mathrm{C}=21 \%$, and $\mathrm{D}=42 \%$. For experiment $\mathrm{B}$, the device was turned upside down to have particles migrate in the opposite direction compared to C. Data suggest not only an amplitude enhancement but also an expected M-shaped feature present in D. (b) Normalised histograms representing a total of 1084 events demonstrating a near 3.5 times enhancement in signal from A to D.

The current profile for individual transit events also reveals striking differences depending on particle trajectory. For particles suspended in the $42 \%$ glycerol solution (buoyant), the current profiles show a clear M-shaped peak, similar to that observed in Fig. 1(a). This effect is present in most events for this data set, and can be postulated a direct result of increased proximity to electrodes. As particles flow over the inner corners of the electrodes, the fringing electric field causes an even greater current change than at the center. These M-shaped pulses are similar to those observed in traditional Coulter counters, present when targets flow too close to the edges of the sensing aperture. ${ }^{11}$

To date, there have been several demonstrations of microfluidic focusing techniques ${ }^{12-15}$ aimed at centering particles within microchannels. In the case of impedance-based volume sensors with coplanar electrodes, the idea is to focus them next to the electrodes. Some examples use focusing 
techniques to improve sensitivity by reducing the sensing volume. One approach is to use sheath flow focusing with an adjustable aperture, ${ }^{16}$ where multiple nonconductive sheaths are used to direct flow of microtargets in conductive media next to a pair of coplanar electrodes in a $\mu$-Coulter counter. Another uses a tunable pneumatic valve to mechanically direct microtargets next to the electrodes. ${ }^{9}$ These approaches allow for increased sensitivities by reducing sensing volumes, but there remains some uncertainty related to variability in particle trajectories above the electrodes. While we demonstrate a simple device that simultaneously enhanced sensitivity and accuracy, we submit that combining buoyancy-based settling (focusing) with these other schemes would further improve on measurement accuracy by confining particles to trajectories that are as close to the coplanar sensing electrodes as possible.

As shown both through FEM modelling and experimental work, focusing microtargets next to the electrodes allows for heightened accuracy and up to a 3.5-fold signal enhancement. The introduced buoyancy-based method is simple to implement and readily combined with current volume sensing technologies. Future work will explore using the M-shaped current pulse to automatically compensate for target height, and thus increase the sensitivity of volume sensors using coplanar electrodes. To increase throughput in future devices, a lengthened meandering channel will allow for beads to flow longer, allowing them to settle more efficiently in rapid flow systems.
The authors thank Dylan Stone for assisting in electrode fabrication and Radin Tahvildari for help in sample preparation. This work is supported by the National Sciences and Engineering Research Council of Canada and the Canadian Foundation for Innovation.

${ }^{1}$ W. H. Coulter, Proc. Natl. Electron. Conf. 12, 1034 (1956).

${ }^{2}$ S. Gawad, L. Schild, and P. Renaud, Lab Chip 1, 76 (2001).

${ }^{3}$ J. Sun, C. C. Stowers, E. M. Boczko, and D. Li, Lab Chip 10, 2986 (2010).

${ }^{4}$ S. Z. Hua and T. Pennell, Lab Chip 9, 251 (2009).

${ }^{5}$ C. Bernabini, D. Holmes, and H. Morgan, Lab Chip 11, 407 (2011).

${ }^{6}$ J. H. Nieuwenhuis, F. Kohl, J. Bastemeijer, P. M. Sarro, and M. J. Vellekoop, Sens. Actuators B 102, 44 (2004).

${ }^{7}$ T. Sun, N. G. Green, S. Gawad, and H. Morgan, IET Nanobiotechnol. 1, 69 (2007).

${ }^{8}$ S. Gawad, K. Cheung, U. Seger, A. Bertsch, and P. Renaud, Lab Chip 4, 241 (2004).

${ }^{9}$ J. Riordon, M. Mirzaei, and M. Godin, Lab Chip 12, 3016 (2012).

${ }^{10}$ M. Godin, F. F. Delgado, S. Son, W. H. Grover, A. K. Bryan, A. Tzur, P. Jorgensen, K. Payer, A. D. Grossman, M. W. Kirschner, and S. R. Manalis, Nat. Methods 7, 387 (2010).

${ }^{11}$ T. Allen, Particle Size Measurement, 5th ed. (Springer, 1996), Vol. 1.

${ }^{12}$ J. Zhu, T.-R. Tzeng, G. Hu, and X. Xuan, Microfluid. Nanofluid. 7, 751 (2009).

${ }^{13}$ L.-M. Fu, C.-H. Tsai, and C.-H. Lin, Electrophoresis 29, 1874-1880 (2008).

${ }^{14}$ J. P. Golden, J. S. Kim, J. S. Erickson, L. R. Hilliard, P. B. Howell, G. P. Anderson, M. Nasir, and F. S. Ligler, Lab Chip 9, 1942 (2009).

${ }^{15}$ S. Choi and J.-K. Park, Anal. Chem. 80, 3035 (2008).

${ }^{16}$ R. Rodriguez-Trujillo, O. Castillo-Fernandez, M. Garrido, M. Arundell, A. Valencia, and G. Gomila, Biosens. Bioelectron. 24, 290 (2008). 